\title{
Algorithm for processing dispersive waves from dipole sonic tool
}

\section{Maurício Redaelli ${ }^{1}$, Rodolfo A. Victor ${ }^{1}$, Carlos F. Beneduzi ${ }^{1}$, Pablo D. Batista ${ }^{2}$}

${ }^{1}$ Petrobras/CENPES; ${ }^{2} \mathrm{CBPF}$

Copyright 2019, SBGf - Sociedade Brasileira de Geofísica

This paper was prepared for presentation during the $16^{\text {th }}$ International Congress of the Brazilian Geophysical Society held in Rio de Janeiro, Brazil, 19-22 August 2019.

Contents of this paper were reviewed by the Technical Committee of the $16^{\text {th }}$ International Congress of the Brazilian Geophysical Society and do not necessarily represent any position of the SBGf, its officers or members. Electronic reproduction or storage of any part of this paper for commercial purposes without the written consent of the Brazilian Geophysical Society is prohibited.

\section{Abstract}

Shear sonic wave velocities can be obtained by monopole sources in logging tools only for fast formations, namely where formation shear velocity is higher than wave velocity in the drilling mud. The dipole source is used for estimating shear velocity in slow formations, by indirectly processing the flexural mode excited in the borehole. The dispersive characteristic of the flexural mode, nevertheless, renders useless the Slowness-Time Coherence method, the traditional algorithm used for processing non-dispersive sonic waveforms from monopole sources. We report the application of Dispersive Slowness-Time Coherence to process sonic log waveforms acquired using dipole sources. This method uses modeled dispersive curves created from classic wave propagation theory and can overcome the previously mentioned limitations. We tested our implementation using real data from four wells drilled in formations with distinct elastic characteristics and found our results compatible to the ones offered by service companies.

\section{Introduction}

Processed sonic log waveform raw data is the main source of information for compressional and shear wave velocities, two parameters that compose every basic dataset used for petrophysical evaluation of hydrocarbon reservoirs. Sonic velocities can be used in porosity and elastic properties estimations, seismic time-depth calibration, besides investigation of anisotropy and hydraulic permeability. One key constraint in processing sonic waveforms is obtaining shear wave velocities in slow formations, which is usually done indirectly by processing the highly dispersive flexural modes excited in the borehole by dipole sources in the logging tool. These products are usually offered by commercial software from service companies, but their processing algorithms are often not clear to the final user, allowing little or no control of the outcomes. In this work we implemented and successfully tested one generalization of the traditional coherence-based processing method, aiming to develop an independent solution from those offered by services companies.

The main method for processing sonic waveforms generated by monopole sources is the Slowness-Time
Coherence (STC). It's a reliable method for processing the non-dispersive headwave modes generated by this kind of source, providing sonic logs for both $V_{P}$ and $V_{S}$ when the formation is fast. However, for slow formations, namely where $V_{S}$ is smaller than the wave velocity in the drilling mud, the monopole source can probe only $V_{P}$. Industry overcame the limitation by introducing the dipole source in the sonic tool. This source generates a flexural mode in the borehole, for which low-frequency components travel with velocity close to formation $V_{S}$. Nevertheless, this behavior raises the need for another processing method, once STC is not suitable for handling dispersive data.

To overcome this limitation, we implemented the Dispersive Slowness-Time Coherence (DSTC) to process dipole waveforms. DSTC uses dispersion curves in the Fourier domain to correct for the dispersive effects found in the dipole waveforms, then processing the resulting pulses using STC. The success of this method depends on the use of suitable dispersion curves, which we obtained by theoretical wave propagation modeling.

\section{Methods}

The STC method (Kimball and Marzetta, 1984) is based on a coherence estimation by cross-correlating the acquired waveforms. The coherence $\rho(T, s)$ is defined by

$$
\rho(T, s)=\frac{\int_{T}^{T+T_{w}}\left|\sum_{m=1}^{N} X_{m}[t+s(m-1) d]\right|^{2} d t}{N \int_{T}^{T+T_{w}} \sum_{m=1}^{N}\left|X_{m}[t+s(m-1) d]\right|^{2} d t}
$$

where $T$ is the reference time, $T_{w}$ is the length of the integration window, $N$ is the number of receivers in the logging tool, $X_{m}$ is the $m-t h$ waveform, $s$ if the wave slowness (reciprocal of velocity), and $d$ is the distance between receivers. Eq (1) shifts the $m-t h$ waveform by an amount of time defined by the guessed slowness $s$ and the distance $d(m-1)$ between the $m-t h$ receiver and the first receiver.

For every position in the time axis and every slowness in the vertical axis, this method computes a coherence value that can be shown in a STC map or a contour plot. We highlighted in Eq. (1) the time shift term in gray and the 
stacking term in green. Wherever the map shows a coherence peak, one can extract from the axis the slowness and the time of occurrence of one wave mode propagation, e.g. a P-wave or an S-wave. The timewindow allows for the separation of events in the STC calculation. Figure 1 shows a coherence map with two peaks representing the coherence of $P$ and $S$ events for one single depth, with arrows pointing the slowness' values read in the map and the corresponding final sonic log positions.

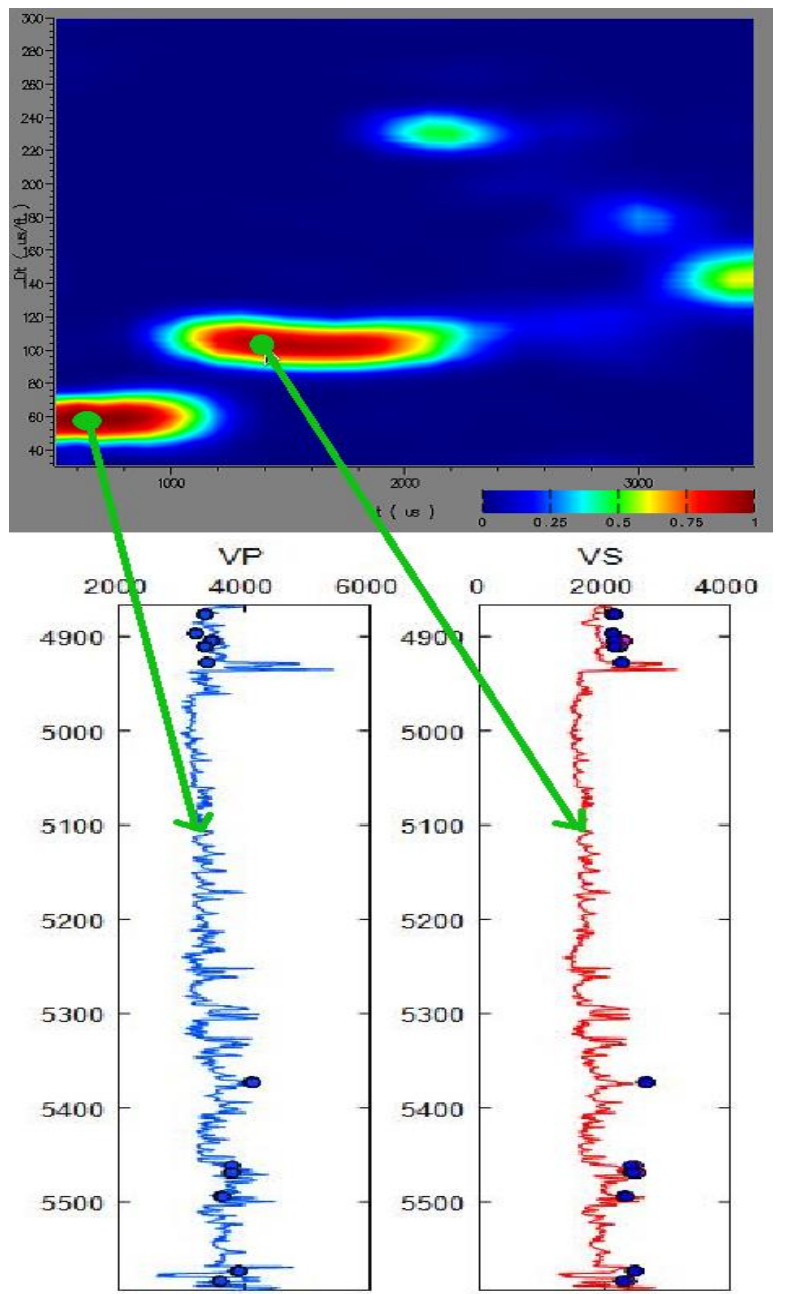

Figure 1: Top: STC map showing two coherence peaks for two different wave modes, and arrows pointing their values in the sonic log depth (bottom).

Dispersive modes are a major limitation for using STC, because the method may find multiple slowness values that maximize the coherence in very close positions in the time axis. Moreover, the weak flexural mode excitation in the low-frequency range lowers the signal-to-noise ratio in the region of interest, reducing the final quality of shear wave velocity estimation through STC. Figure shows an STC plot computed for dipole data. The region of maximum coherence is distorted and ultimately useless for accurate estimation of $V_{S}$. To circumvent this problem, Kimball (1998) proposed the DSTC method.

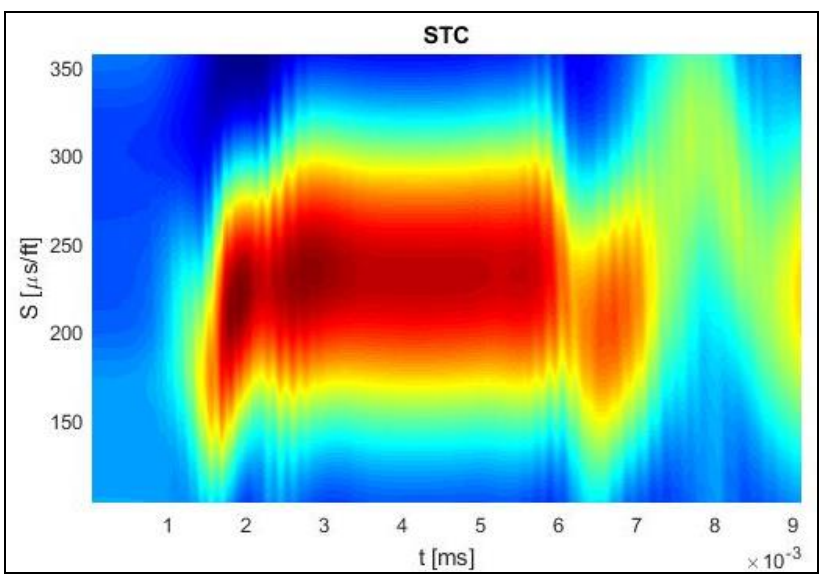

Figure 2: STC plot computed for dipole data. There are more than one possible slowness values competing for the final result. The coherence peaks tend to be distorted when STC is used to process dipole data.

The DSTC is based on the time-shift property of the Fourier Transform, where a shift in the time domain corresponds to a linear phase shift in the frequency domain. Thus, the time shift that would be applied by the STC (gray in Eq. 1) becomes a multiplication of the transformed waveforms by a phase-factor:

$$
X_{i}[t-s(m-1) d]=\mathcal{F}^{-1}\left[X_{i}(f) e^{-j 2 \pi f s(m-1) d]}\right]
$$

where $F^{-1}$ denotes the inverse Fourier Transform, $f$ is the frequency, and $j=\sqrt{-1}$ is the imaginary unit.

The DSTC algorithm first performs a Fourier Transform in each waveform, subsequently applying the phase correction as in Eq (2), but now the slowness defining the phase shift is also a function of frequency, defined by a dispersion curve. In other words, before returning the transformed waveforms to the time domain, the DSTC method corrects the dispersion effect using dispersioncurves that are functions of the slowness. After the correction and the inverse Fourier Transform, the method is similar to the non-dispersive STC.

Figure 3 shows in the left panel 13 synthetic waveforms with non-dispersive waves (colors) and dispersive waves (black). Due to the dispersion, the waveforms change shape gradually as the wave propagates from the first to the last receiver. The top-left panel shows the average amplitude spectrum in blue and dispersion curve in red. The dispersive waveforms were built using this dispersion curve, while the non-dispersive waveforms were built using the low-frequency slowness value, of $190 \mu \mathrm{s} / \mathrm{ft}$. The bottom-left panel shows individual amplitude spectra (black) and phase spectra (red). 


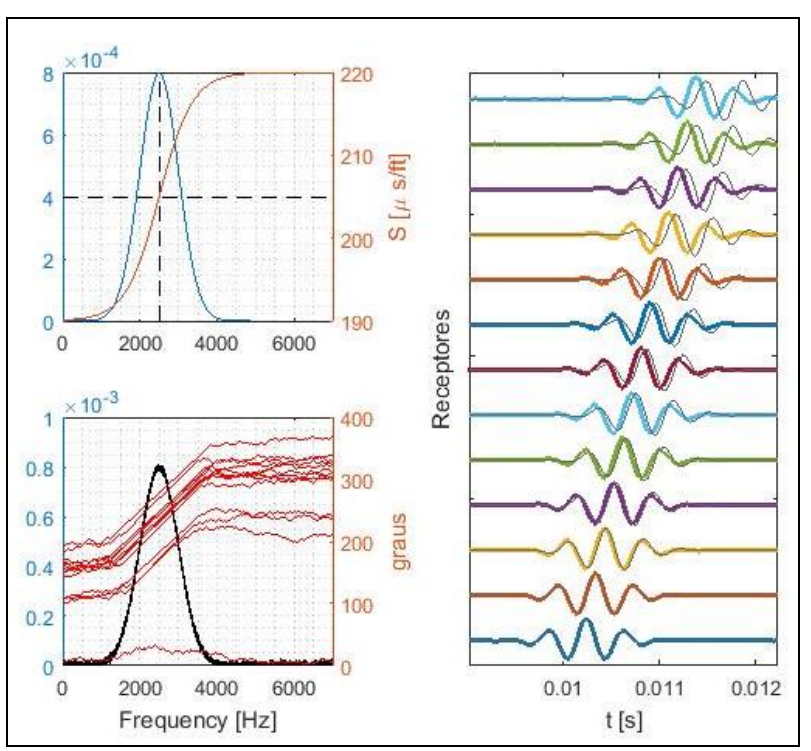

Figure 3: Right: synthetic waveforms of 13 receivers for non-dispersive waves (colors) and dispersive waves (black). Top-left: average amplitude spectrum (blue) and dispersion curve (red). Bottom-left: individual amplitude spectra (black) and phase spectra (red).

Figure 4 shows the processing results for the two methods: STC in the left panel and DSTC in the right panel, for the synthetic data shown in Figure 3. The STC map shows a peak around $205 \mu \mathrm{s} / \mathrm{ft}$. One can verify that this value is close to the slowness at maximum amplitude in the spectrum (see Figure 3). The DSTC map, meanwhile, shows a peak around $190 \mu \mathrm{s} / \mathrm{ft}$, in agreement to the low-frequency slowness. This preliminary result confirms that the DSTC can correct the dispersion in the waveforms.

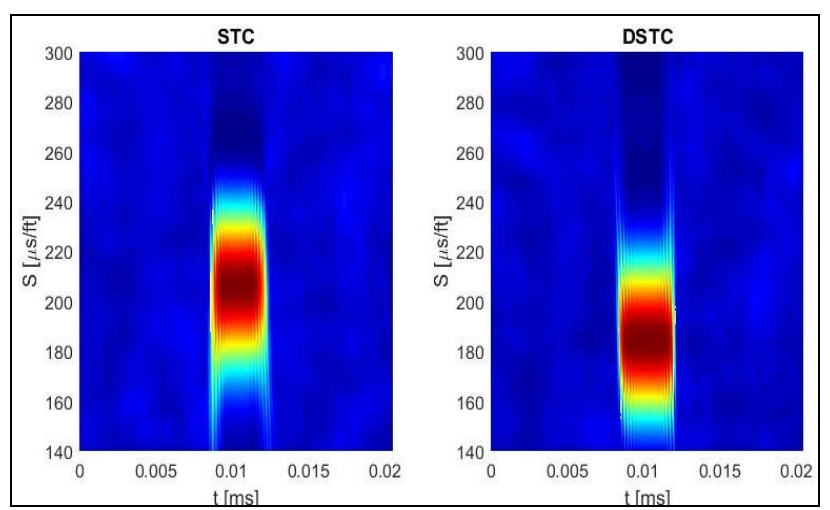

Figure 4: Left: STC map with a peak around $205 \mu \mathrm{s} / \mathrm{ft}$. Right: the DSTC map with a peak around $190 \mu \mathrm{s} / \mathrm{ft}$, in agreement to the low frequency slowness.

One key point for using DSTC is obtaining dispersion curves that represent the dispersion observed in the waveforms. For this, we used a model based on the classic wave propagation theory. It is a solution for the homogeneous form of the Navier equation (Tang \& Cheng, 2004), describing the displacements in the borehole system where normal modes of propagation are excited.

Our model is a function of the formation parameters, such as compressional and shear formation velocities and formation density, and borehole parameters, namely radius, drilling mud density, and drilling mud velocity. A dispersion curve comes from the roots of the determinant of the coefficient matrix formed from the system of equations generated by applying borehole boundary conditions to the Navier equations. In order to generate a dispersion curve, a root finding procedure must be evaluated for all possible frequencies. Figure 5 shows the determinant for the $1600 \mathrm{~Hz}$ frequency with one root (red circle) that is the flexural wave velocity for this frequency. The flexural mode must be slower than the shear wave mode in the formation (Tang \& Cheng, 2004), so the procedure neglects every root at the right of the red vertical line in Figure 5.

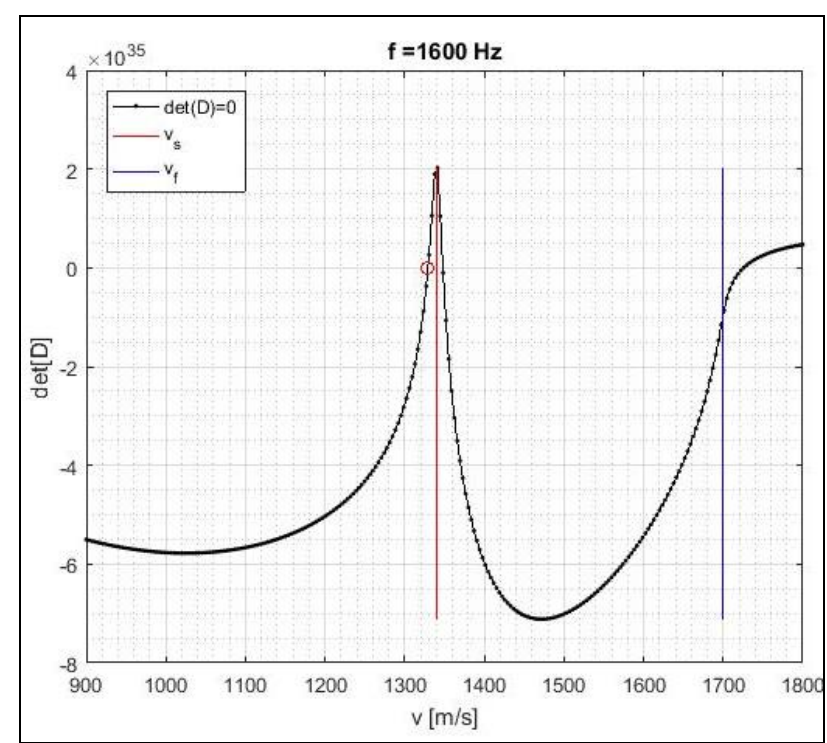

Figure 5: Determinant for the $1600 \mathrm{~Hz}$ frequency with one root (red circle): the flexural wave velocity for this frequency.

Figure 6 shows a dispersion curve obtained for a set of borehole and formation parameters (blue line), the amplitude spectrum (yellow dashed line) and the dispersion points observed in real dipole waveforms (dots with colors according to the amplitude spectrum), the last obtained by Phase Based Dispersion Analysis (PBDA) proposed by Assous (2014). Even though the PBDA method delivers data-driven dispersion estimates, flexural mode amplitude spectrum in slow formations usually shows a very low signal-to-noise ratio, making it difficult to obtain the shear wave velocity from extrapolating experimental data. This lack of signal in low frequencies, the most important region of the spectrum for dipole sonic log processing, is the main reason we chose the modeldriven approach, such as DSTC.

Finally, for DSTC, all the model parameters must be held constant, except the shear velocity, that must be varied in a pre-defined range, in order to check which dispersion curve maximizes the semblance between the waveforms. 


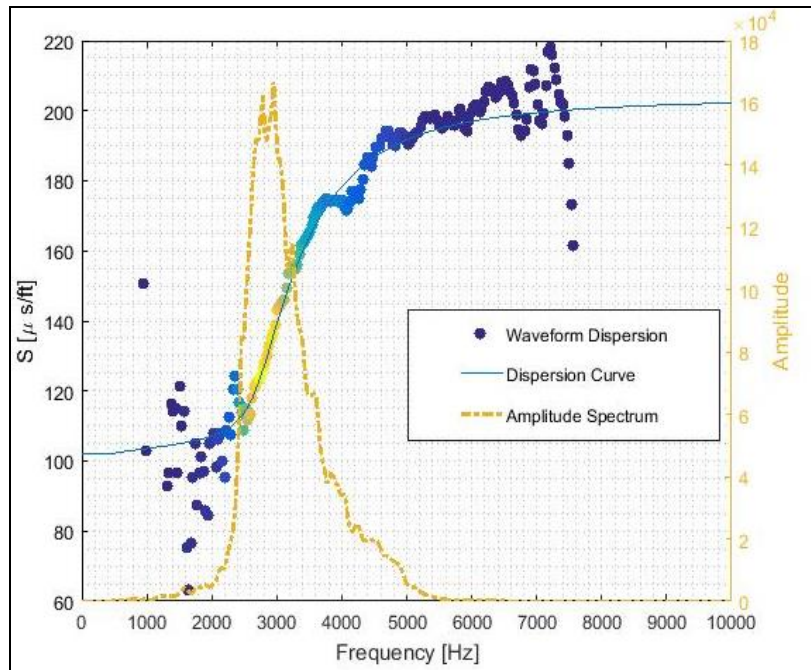

Figure 6: Dispersion curve (blue line), amplitude spectrum (yellow dashed line) and the dispersion observed in real dipole waveforms (dots with colors according to the amplitude in the spectrum) obtained by the PBDA method.

\section{Results}

We tested our implementation for processing real data from four wells with different characteristics. Well A dipole data was acquired in a fast formation region and was processed using both STC and DSTC. Figure 7 shows in the left track of both panels the resulting log (green line) against the reference sonic log, processed using commercial software (white line), with the semblance map collapsed to the slowness axis direction in the background. We also show the difference plot between our results and the reference log. The left panel shows these features for the DSTC, while right panel shows them for the STC. Figure 7 confirms that STC is not suited for dipole data, overestimating the slowness in the entire range. It also shows that DSTC can process dipole data much more accurately, producing an unbiased difference log with small deviations.

Figure 8 shows a similar plot for the Well B, with data acquired in a slow formation region. Although in this case the STC performed better than in Well A, it still shows a bias in the difference plot, suggesting that the method overestimates the slowness values. The DSTC produces better results.

Figure 9 shows results for Well $\mathrm{C}$ with data from a very slow formation, plotted against the reference log (white line) and with the coherence map collapsed to the slowness direction in the background. DSTC performed very well, with small differences for the reference result.

Finally, Figure 10 shows results for Well D, in a slow formation with a non-uniform caliper. Such cases are challenging for processing, even using software from service companies, as a uniform borehole is assumed in the wave propagation theory. Nevertheless, we consider the results satisfactory, with a small overestimation in the slowness only in the non-uniform diameter region.

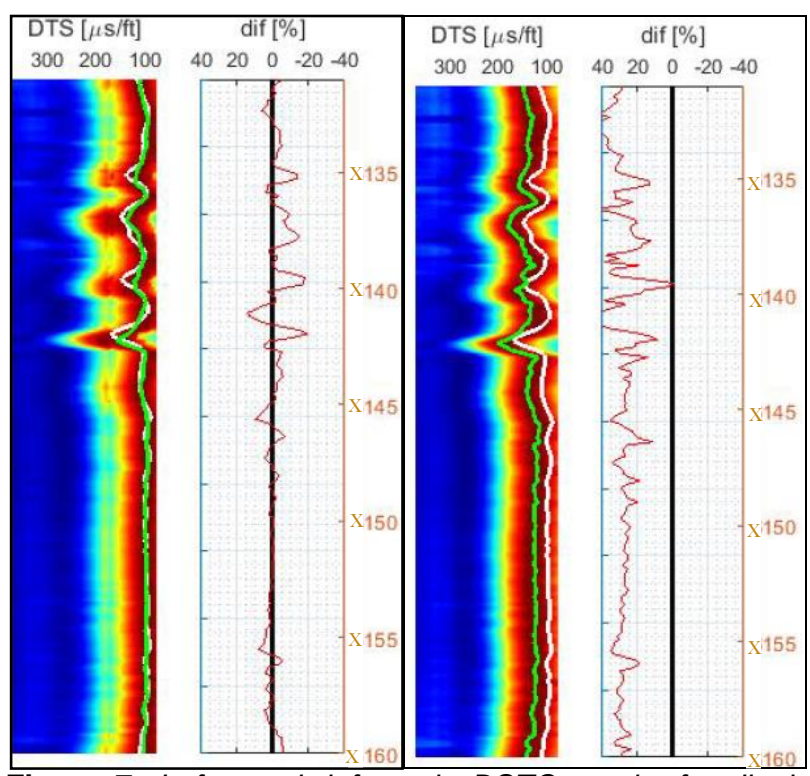

Figure 7: Left panel, left track: DSTC results for dipole data of Well A (green line), plotted against the reference log (white line) and with the coherence map collapsed to the slowness direction in the background. Left panel, right track: difference log, computed between our results and the reference log. This result shows a difference log with low deviations, which confirms that the DSTC is capable of correcting the dispersion in the dipole data. Left panel: the same layout as the left panel, but using STC. This method clearly overestimates de slowness values.

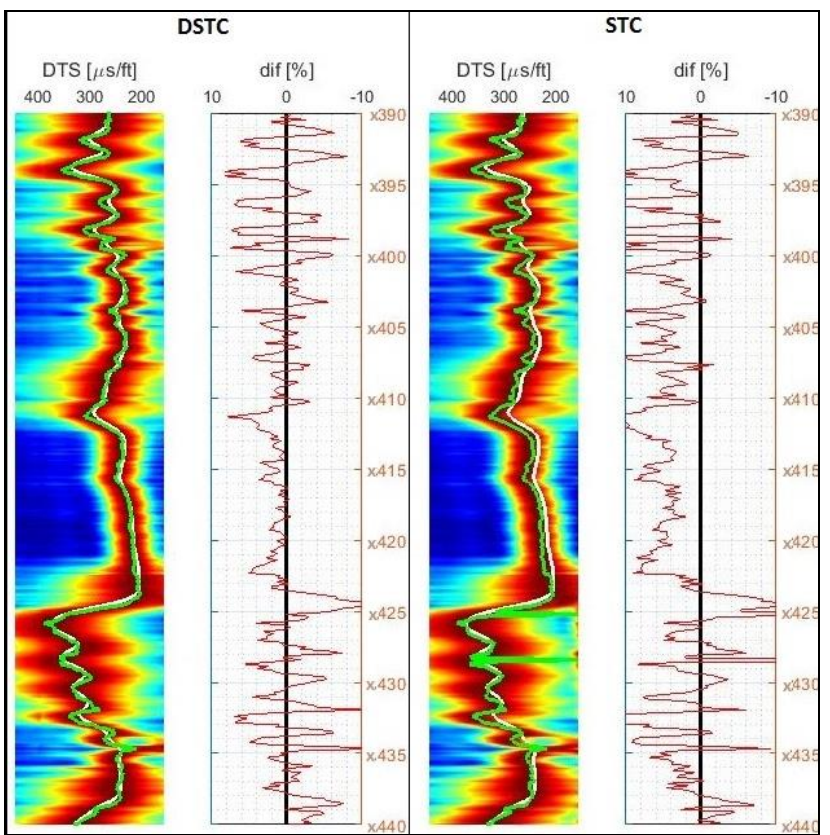

Figure 8: Well B slow formation processing, in the same layout as Figure 7. Although STC performed better than in Well $A$, it still shows an overestimating bias in the difference plot. DSTC produces better results. 


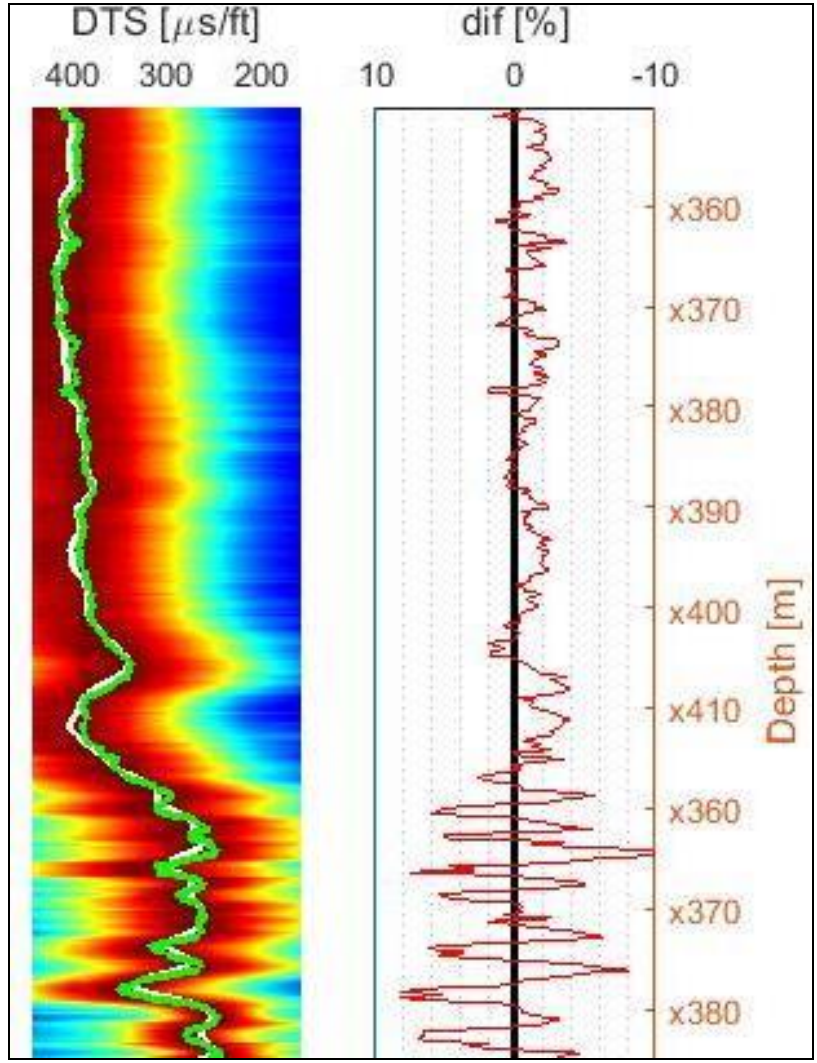

Figure 9: left track, the DSTC method results for dipole data of Well $C$ (green line), plotted against the reference log (white line) and with the coherence map collapsed to the slowness direction in the background. Right track: difference log, computed between our results and the reference log.

\section{Conclusions}

Shear wave slowness from sonic log in slow formations can be obtained from modeling flexural waves. We demonstrated that non-dispersive methods (STC) are not suitable for extract correct shear slowness values from this wave mode, and implemented DSTC that corrects the dispersion effects using theoretical dispersion curves. We also built an algorithm based on classical wave theory to obtain the dispersions curves from borehole and formation parameters, to be used as an input for the DSTC method.

The results are far better than those produced using the STC method. We successfully processed four sonic logs and compared them to reference logs coming from commercial software or service companies. Our results are similar to the reference ones.

The theoretical model used in this work assumes a homogenous, isotropic media, with source and receivers in the exact center of an open, vertical borehole and constant caliper. These assumptions simplify the math, but can negatively impact the solutions in more complex formations or damaged wellbore walls. For further advanced studies, we intend to further improve the model in order to account for anisotropic formations, irregular borehole profile, the presence of casing and tool, LWD acquisitions and deviated wells.

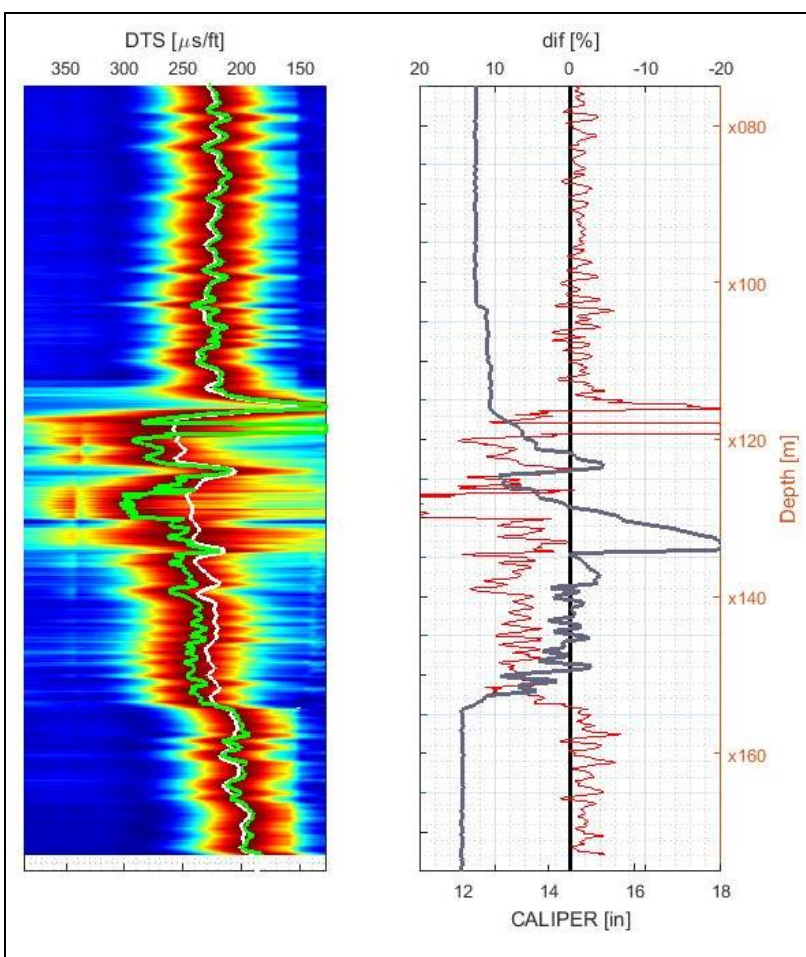

Figure 10: Results for Well $D$ results. The right track shows the caliper log (gray), with a non-uniform borehole diameter between $\sim 2120 \mathrm{~m}$ and $\sim 2150 \mathrm{~m}$. In this region, the processing methods can struggle, as they assume constant diameter. The results, shown in the left panel, are worse in this region. Still, the differences are small.

\section{Acknowledgments}

We thank Tiago de Bittencourt Rossi for the support with data and processing, and Petrobras for providing the data and the permission for publication.

\section{References}

KIMBALL, C. V.; MARZETTA, T. L. Semblance processing of borehole acoustic array data. GEOPHYSICS, v. 49, n. 3, p. 274\{281, mar. 1984.

KIMBALL, C. V. Shear slowness measurement by dispersive processing of the borehole fexural mode. GEOPHYSICS, v. 63, n. 2, p. 337\{344, mar. 1998.

TANG, X. M.; CHENG, C. H. A. Quantitative borehole acoustic methods. 1st ed. ed. Amsterdam ; Boston: Elsevier, 2004. (Handbook of geophysical exploration. Seismic exploration, v. 24). OCLC: ocm54610927.

ASSOUS, S.; ELKINGTON, P.; LINNETT, L. Phasebased dispersion analysis for acoustic array borehole logging data. The Journal of the Acoustical Society of America, v. 135, n. 4, p. 1919\{1928, abr. 2014. 